\title{
RADIO CONTINUUM SURVEYS ABOVE 2 GHZ
}

\author{
J.L. JONAS
}

Department of Physics and Electronics, Rhodes University

\section{Current and Future Surveys}

The Rhodes/HartRAO $2326 \mathrm{MHz}$ radio continuum survey was observed at HartRAO over a 13 year period (Jonas et al. 1985, Mountfort et al. 1987, Jonas \& Baart 1995, Jonas et al. 1996). This is the highest frequency and highest angular resolution (HPBW $=20^{\prime}$ ) all-sky radio continuum survey made using a ground-based telescope. The $1 \sigma$ noise level is better than $30 \mathrm{mK}$. A high-pass filtered image of the data is shown in Figure 1.

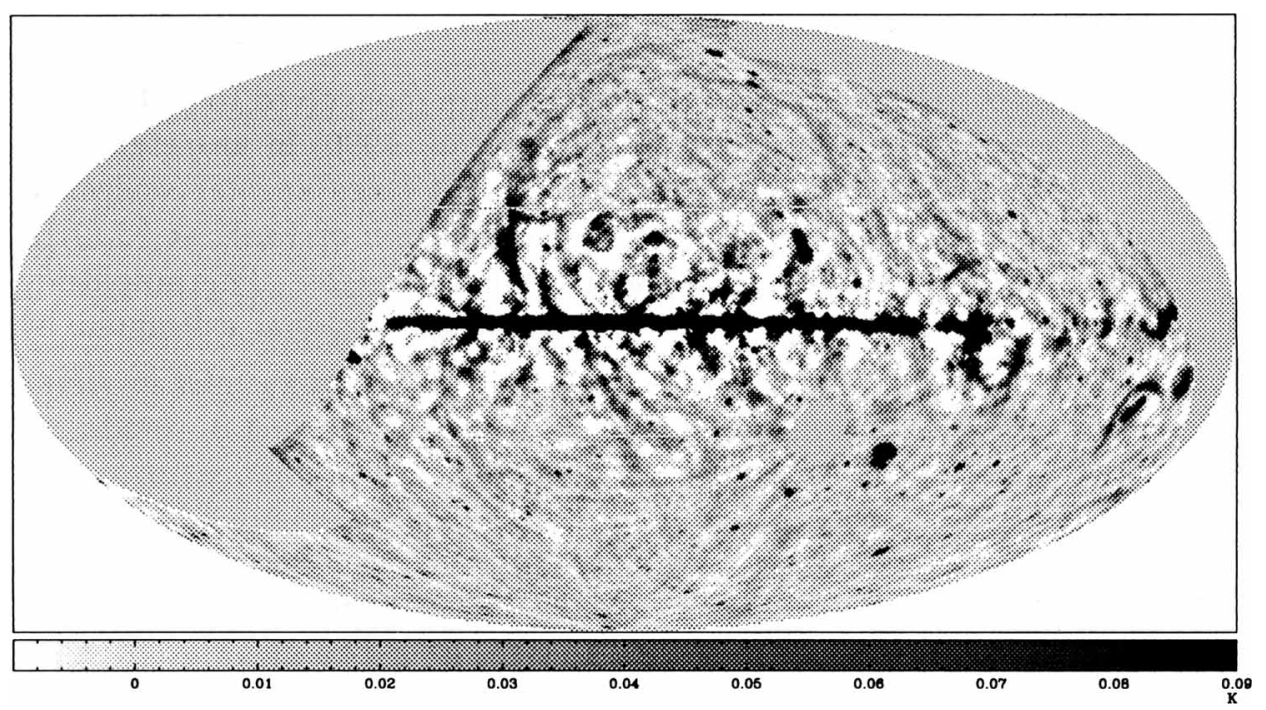

Figure 1. A high-contrast image of the Rhodes/HartRAO $2326 \mathrm{MHz}$ radio continuum survey after processing with a median high-pass filter which removed structures with a scale size greater than $3^{\circ}$. 
The scientific competence of ground-based, single-dish radio continuum survey data at $\mathrm{GHz}$ frequencies is constrained by the following environmental realities and experimental difficulties: (a) Contamination from ground radiation and atmospheric absorption makes absolute temperature measurements difficult and contributes to baseline uncertainties. (b) The increasing number of sources of radio frequency interference (terrestrial and satellite) are making ground-based radio continuum measurements impossible in certain bands. (c) Receivers with exceptional gain and noise temperature stability are required for large area surveys. (d) Temperature scale calibration is difficult for large dish antennae. These factors lead to systematic errors in the survey data, specifically "scanning effects," false large-scale structures and temperature calibration errors.

We have constructed a small, portable $2.3 \mathrm{GHz}$ horn telescope with which we intend to make a low-resolution $\left(\mathrm{HPBW}=15^{\circ}\right)$ survey of the southern sky. The results from this experiment will be used to improve the calibration of the main survey.

We have made observations of three regions of the Galactic plane at $8400 \mathrm{MHz}$ using the HartRAO telescope (e.g., du Plessis et al. 1995) to determine the feasibility of an extensive survey of the southern Galactic plane. The results of these observations were encouraging, and when the HartRAO $8400 \mathrm{MHz}$ receiver has been upgraded to support dual polarization we will pursue this new survey project.

\section{COBE DMR Comparisons}

We have cross-correlated the $2.3 \mathrm{GHz}$ survey data (convolved to $7^{\circ} \mathrm{HPBW}$ ) with the 4-year COBE DMR data (Bennett et al. 1996) in an attempt to quantify the Galactic foreground contribution at large angular scales. A preliminary investigation yields an RMS contribution of $13 \mu \mathrm{K}$ at $53 \mathrm{GHz}$, which corresponds to a temperature spectral index of -2.7 . This amplitude is somewhat larger than the Galactic synchrotron residual estimated by Kogut et al. (1996).

\section{References}

Bennett,C.L., et al. 1996, Astrophys.J. 464, L1

du Plessis,I., et al. 1995, Astrophys.J. 453, 746

Jonas,J.L., de Jager,G. \& Baart,E.E., 1985, Astron.Astrophys.Supp. 62, 105

Jonas,J.L. \& Baart,E.E., 1995, Astron.Astrophys.Supp. 230, 351

Jonas,J.L., Baart,E.E. \& Nicolson,G.D., 1996, Mon.Not.R.astron.Soc. in preparation

Kogut,A., et al. 1996, Astrophys.J. 464, L5

Mountfort,P.I., et al., 1987, Mon.Not.R.astron.Soc. 226, 917 